\title{
Analysis of common type 2 diabetes mellitus genetic risk factors in new-onset diabetes after transplantation in kidney transplant patients medicated with tacrolimus
}

\author{
Mateusz Kurzawski • Krzysztof Dziewanowski • \\ Joanna Lapczuk • Anna Wajda • Marek Droździk
}

Received: 2 February 2012 / Accepted: 10 April 2012 /Published online: 9 May 2012

(C) The Author(s) 2012. This article is published with open access at Springerlink.com

\begin{abstract}
Purpose New-onset diabetes after transplantation (NODAT) is a major complication after kidney transplantation. The risk factors for NODAT include the use of calcineurin inhibitors as part of the immunosuppressive regimen, among which tacrolimus has the most pronounced diabetogenic effect. Both NODAT and type 2 diabetes mellitus (T2DM) share several risk factors. Recent studies have identified a number of common genetic variants associated with increased risk of T2DM. Here we report the results of our study on the potential effect of single nucleotide polymorphisms (SNPs) previously associated with T2DM on the risk of NODAT in kidney transplant patients medicated with tacrolimus.

Methods Seven SNPs in six genes known to increase the risk of T2DM in Caucasians were genotyped by means of TaqMan assays in 235 kidney transplant patients medicated with tacrolimus: rs4402960 and rs1470579 in IGF2BP2; rs1111875 in HHEX; rs10811661 upstream of $C D K N 2 A / B$; rs13266634 in SLC30A8; rs1801282 in PPARG; rs5215 in KCNJ11. The TCF7L2 rs7903146 SNP was also included in the multivariate analysis.

Results None of the analyzed SNPs was significantly associated with the risk of NODAT. However, the IGF2BP2
\end{abstract}

M. Kurzawski $(\bowtie) \cdot J$. Lapczuk · A. Wajda $\cdot$ M. Droździk

Department of Experimental and Clinical Pharmacology,

Pomeranian Medical University,

Powstancow Wlkp. 72,

70-111 Szczecin, Poland

e-mail: mkurz@op.pl

K. Dziewanowski

Clinical Department of Nephrology and Dialysis,

Marie Curie Regional Hospital,

Arkonska 4,

71-455 Szczecin, Poland rs4402960 T allele was present significantly more frequently among patients diagnosed with NODAT more than 2 weeks after transplantation $(p=0.048)$. Mean $( \pm$ standard deviation $)$ number of the analyzed alleles tended to be lower in patients without NODAT $(6.19 \pm 1.71)$ than in NODAT patients $(6.58 \pm 1.1 .95 ; p=0.09)$ and significantly lower compared to late-onset NODAT patients $(7.03 \pm 1.88 ; p=0.018)$. Multivariate analysis confirmed the significance of 'diabetogenic' allele number in late-onset NODAT development [odds ratio (OR) 1.37, $95 \%$ confidence interval (CI) 1.05-1.78; $p=0.017]$. Additionally, individuals carrying $>7$ of the analyzed 'diabetogenic' alleles were at a significantly higher risk of NODAT (OR 2.17, $95 \%$ CI 1.18-3.99; $p=0.015$ ). Conclusions Complex analysis of genotypes increasing the risk of diabetes may lead to the identification of NODAT susceptibility predictors.

Keywords NODAT $\cdot$ Kidney transplantation $\cdot$ Single nucleotide polymorphisms · Type 2 diabetes mellitus

\section{Introduction}

New-onset diabetes after transplantation (NODAT) is one of the major complications after kidney transplantation. The term 'NODAT' has replaced the older term 'post-transplant diabetes mellitus' (PTDM) to differentiate new-onset diabetes from diabetes developed prior to transplantation [1]. It is now widely accepted that NODAT leads to graft failure and promotes cardiovascular disease, the main cause of death in kidney transplant recipients [2]. The incidence of NODAT in patients after solid organ transplantation reported in a large meta-analysis ranged from 2 to $50 \%$ at 1-year posttransplantation [3]. The pathophysiology of NODAT closely mimics that of type 2 diabetes mellitus (T2DM), with both 
diseases characterized by a combination of insulin resistance and insulin hyposecretion. However, insulin hyposecretion has been a key determinant of worsening glucose tolerance following renal transplantation [2]. The risk factors for NODAT include both the conventional risk factors for T2DM [e.g., older recipient age, nonwhite ethnicity, family history of diabetes, sedentary lifestyle, high body mass index (BMI), and cytomegalovirus or hepatitis $\mathrm{C}$ virus (HCV) infection] and those specific to transplant patients (acute rejection incidence, high doses of corticosteroids, and an immunosuppressive regimen with calcineurin inhibitors) $[4,5]$.

Among the calcineurin inhibitors used in transplant recipients, the diabetogenic effect of tacrolimus seems to be the most pronounced. In one study, patients receiving tacrolimus had a significantly higher incidence of NODAT than those not medicated with the drug [4]. In another study, patients treated with tacrolimus had a significantly higher incidence of NODAT or impaired fasting glucose after 6 months of therapy than those treated with cyclosporine (33.4 vs. $26.0 \%$, respectively [6]. Tacrolimus has been reported to cause NODAT through decreased insulin secretion of pancreatic beta cells in an animal model, with inhibition of insulin mRNA transcription [7]. Decreased insulin release as a consequence of high tacrolimus concentration was confirmed in human studies; however, insulin resistance was also suggested as a possible mechanism $[8,9]$. An elevated risk of developing NODAT has also been described in prediabetic patients [9]. Hence, NODAT is most probably not a separate entity but a consequence of an underlying glucose metabolic disorder that is uncovered by immunosuppression [5].

Past linkage studies have identified those genes associated with the most prominent T2DM effects, i.e., TCFL7, $P P A R G$, and KCNJ11. More recently genome-wide association studies (GWAS) have confirmed previous reports and uncovered dozens of new genetic variants associated with an increased risk of T2DM [10-15]. A number of the single nucleotide polymorphisms (SNPs) identified to be associated with T2DM are common in Caucasians and may underlie individual susceptibility to NODAT. In some cases an association of T2DM risk alleles with NODAT has been confirmed. A polymorphism of the KCNQ1 gene, which encodes the pore-forming subunit of the voltage-gated $\mathrm{K}+$ channel, has been associated with an increased risk for NODAT in Spanish Caucasians [16], independently of other risk factors. Variants of hepatocyte nuclear family transcription factor 4 alpha $(H N F 4 A)$ and insulin receptor substrate-1 (IRS1) have been associated with NODAT among Hispanic American renal allograft recipients, who are known to have a higher risk of NODAT compared to other Caucasians, despite early steroid withdrawal [17]. An influence of SNPs within the calpain-10 gene (CAPN10) on the occurrence of diabetes in Polish Caucasian kidney transplant recipients has also been described [18]. Additionally, several diabetogenic alleles have been reported to be correlated with NODAT risk in Koreans [19].

The aim of the study reported here was to analyze the potential effect of SNPs previously associated with T2DM on NODAT development in kidney transplant patients medicated with tacrolimus. For the purpose of this study, seven SNPs in six genes, with the frequency of minor allele being $>0.1$ in Caucasians, which had been previously identified and confirmed as 'diabetogenic' in the general population in GWAS studies were selected: rs4402960 and rs1470579 in the $I G F 2 B P 2$ intronic region, rs 1111875 in near HHEX, rs10811661 upstream of $C D K N 2 A / B$, rs13266634 missense in $S L C 30 A 8$, rs 1801282 in the PPARG intron, and rs5215 missense in KCNJ11.

\section{Methods}

\section{Patients}

A total of 235 kidney transplant patients, all Polish Caucasians and non-diabetic at the moment of transplantation (patients with diabetes mellitus prior to the transplantation were excluded) were eligible for enrollment in this retrospective study. Subjects were recruited consecutively from patients who underwent kidney transplantation in the Clinical Department of Nephrology and Dialysis, County Hospital, Szczecin, Poland between 2000 and 2009 and who were subsequently medicated with twice-daily tacrolimus (Prograf; Astellas Pharma, Tokyo, Japan) as a part of immunosuppressive regimen. Patients who did not maintain graft function for at least 1 year post-transplant were excluded. The patients eligible for enrollment were subdivided into two groups: those with NODAT $(n=67)$ and the controls, i.e., without NODAT $(n=168)$. The characteristics of the patients are given in Table 1. Patients with hemoglobin Alc continuously $>6.5 \mathrm{mg} / \mathrm{dL}$, fasting plasma glucose of $>126 \mathrm{mg} / \mathrm{dL}(7.0 \mathrm{nmol} / \mathrm{L})$, or those requiring insulin and/or oral hypoglycemic agents for $>3$ months were diagnosed as having NODAT. NODAT was diagnosed up to 1 year posttransplantation. The observation time was extended in the case of some late-onset NODAT patients in order to achieve the 3-month evaluation period from the onset of diabetes. The NODAT patients were subsequently divided into two groups: those with early-onset NODAT $(n=39)$, in whom diabetes occurred within the first 2 weeks of immunosuppressive therapy, and those with late-onset NODAT, in whom diabetes was found later in the course of treatment $(n=28)$.

The treatment protocol consisted of tacrolimus, mycophenolate mofetil, and steroids. Specifically, tacrolimus 
Table 1 Characteristics of patients enrolled in the study

\begin{tabular}{llll}
\hline Patient characteristics & NODAT $(n=67)$ & No PTDM $(n=168)$ & $p$ \\
\hline Age (years) & $47.7 \pm 10.6$ & $43.2 \pm 13.0$ & $0.014^{\mathrm{b}}$ \\
Sex (female) & $30(45.5)$ & $78(46.4)$ & $1.000^{\mathrm{c}}$ \\
Body mass index & $25.8 \pm 4.1$ & $24.3 \pm 3.7$ & $0.006^{\mathrm{b}}$ \\
Donor age (years) & $47.8 \pm 11.8$ & $46.0 \pm 12.3$ & $0.321^{\mathrm{b}}$ \\
Viral infections & $10(15.2)$ & $27(16.1)$ & $1.000^{\mathrm{c}}$ \\
Acute rejection & $9(13.6)$ & $10(6.0)$ & $0.064^{\mathrm{c}}$ \\
Steroid total dose $(\mathrm{g})$ & $4.20 \pm 1.72$ & $3.76 \pm 2.35$ & $0.017^{\mathrm{d}}$ \\
\hline
\end{tabular}

NODAT, New-onset diabetes after transplantation; PTDM, posttransplant diabetes mellitus

Data are presented as the mean \pm standard deviation, or as the number, with the percentage in parenthesis

${ }^{a}$ Viral infections were: cytomegalovirus (6 vs. 20 in PTDM and control group, respectively), hepatitis C virus (2 vs. 4), and hepatitis B virus (2 vs. 3)

${ }^{\mathrm{b}}$ Student $t$ test

${ }^{\mathrm{c}}$ Fisher exact test

${ }^{\mathrm{d}}$ Mann-Whitney $U$ test

therapy was initiated at $0.1 \mathrm{mg} / \mathrm{kg} /$ day with doses adjusted to maintain trough levels of between 10 and $12 \mathrm{ng} / \mathrm{mL}$ in the first month post-transplantation, and then between 8 and $10 \mathrm{ng} / \mathrm{mL}$. Whole blood tacrolimus concentration was assessed with the use of a chemiluminescent microparticle immunoassay (CMIA; Architect Tacrolimus Assay, Abbott, Germany). An initial oral dose of mycophenolate mofetil $2.0 \mathrm{~g} /$ day was administered once daily or given in equally divided doses every $12 \mathrm{~h}$. Methylprednisolone was given concomitantly: a dose of $500 \mathrm{mg}$ on the day of surgery, tapered to $40 \mathrm{mg}$ /day during the first week, followed by $30 \mathrm{mg} /$ day of prednisolone in the second week, $20 \mathrm{mg} /$ day of prednisolone in the third week, $15 \mathrm{mg}$ /day in the fourth week, and $10 \mathrm{mg} /$ day thereafter. Total corticosteroid dose for each patient during the first year of the study was calculated, and methylprednisolone was recalculated to prednisolone using $r=1.25$ cofactor. All patients gave written informed consent to participate in the study, and a relevant ethics committee approved the study protocol.

\section{Genotyping}

Genomic DNA was extracted from $200 \mu \mathrm{L}$ of whole blood samples using the GeneMATRIX Quick Blood DNA Purification kit (EURx, Gdansk, Poland). Pre-validated allelic discrimination TaqMan real-time PCR assays (Applied Biosystems, Foster City, CA) were used to detect SNPs previously associated with T2DM in the general population. The following SNPs in their respective genes were analyzed (assay IDs are given in parentheses): rs4402960 (C 2165199 10) and rs1470579 (C 2165184 10) in $I G F 2 B P 2$, rs 1111875
(C 11214581 10) in $H H E X$, rs 10811661 $\left(\mathrm{C}_{-}^{-} 31288917,10\right)$ in $C D K N 2 A$, rs 13266634 $\left(\mathrm{C}_{--}^{-} 357888_{-}^{-} 10\right)$ in SLC30A8, rs 1801282 $\left(\mathrm{C}_{---} 1129864,10\right)$ in PPARG, and rs 5215 (C_2991148_10) in KCNJ11. Fluorescence data were captured using an ABI PRISM 7500 FAST Real-Time PCR System (Applied Biosystems) after 40 cycles of PCR.

\section{Statistical analysis}

Categorical variables (i.e., allele, genotype, haplotype frequencies, acute rejection episodes) were compared by the Fisher exact test and chi-square test. Odds ratios (OR) and $95 \%$ confidence intervals $(95 \% \mathrm{CI})$ were calculated using the Newcombe-Wilson method without the continuity correction. Multivariate logistic regression model was used to test independent NODAT risk factors. Variables identified as potentially significant $(p<0.1)$ were included in multivariate analysis (age, BMI, acute rejection episodes, total steroid dose) together with the sum of alleles previously associated with increased risk of T2DM in the general population: IGF2BP2 rs4402960 T, HHEX rs1111875T, CDKN2A rs $10811661 \mathrm{C}, S L C 30 A 8$ rs $13266634 \mathrm{C}, P P A R G$ rs $1801282 \mathrm{C}$, and KCNJ11 rs5215 C. The TCF7L2 rs7903146 $\mathrm{T}$ allele was added to the multivariate analysis, as genotyping data were available from our previous study for all patients [20] and this SNP has also been associated with T2DM [21]. IGF2BP2 rs $1470579 \mathrm{C}$ was not further analyzed, as it was found to be in almost complete linkage disequilibrium with $I G F 2 B P 2 \mathrm{rs} 4402960 \mathrm{~T}\left(D^{\prime}=1.0, r^{2}=\right.$ 0.981), forming a common haplotype that includes minor alleles for both loci. Hence, it could not be considered as an independent variable. In each patient, the number of 'diabetogenic' alleles was calculated for a given SNP (i.e., 0, 1, or 2 ), and values for all SNPs were subsequently summarized to obtain a total number of 'diabetogenic' alleles. A $p$ level of $<0.05$ was considered to be statistically significant. The data were tested for their fit to Hardy-Weinberg equilibrium by calculating expected frequencies of genotypes and comparing them to the observed values using a chi-square test. All calculations were performed using the Statistica 9.0 software package (Statsoft, Tulsa, OK).

\section{Results}

Mean patient's age, BMI, and total steroid dose were higher in the NODAT group than in the non-NODAT patients (controls). No differences in gender, donor age, viral infection frequency, nor acute rejection episodes were noted between these two groups (Table 1). In terms of genotype frequency distribution, none of the SNPs analyzed showed a significant deviation from the Hardy-Weinberg equilibrium. 
None of the analyzed SNPs was significantly associated with the risk of NODAT (Table 2). However, of the seven SNPs analyzed, six (with the exception for KCNJ11 rs5215) alleles previously identified as T2DM risk factors occurred more frequently in the NODAT group than in patients who did not develop diabetes (difference was not significant). The NODAT patients were subsequently divided into those with early-onset NODAT $(n=39)$ and late-onset NODAT $(n=28)$, respectively. No association between genetic factors and early-onset NODAT was observed, although the IGF2BP2 rs4402960 T allele was found significantly more frequently among patients diagnosed with NODAT later than 2 weeks post-transplantation $(p=0.048$; Table 2$)$. Of the seven analyzed 'diabetogenic' alleles, six were more frequent in the late-onset NODAT patients than in the patients without diabetes (exception: the PPARG rs1801282 C allele). Due to the small number of patients in the subgroups, these differences were not significant. In order to investigate a cumulative effect of SNPs previously associated with T2DM in relation to NODAT, we analyzed the total number of 'diabetogenic' alleles inherited by each patient. The mean number of analyzed alleles tended to be lower in patients without NODAT $(6.19 \pm 1.71)$ than in all NODAT patients $(6.58 \pm 1.1 .95 ; p=0.09)$ and to be significantly reduced in comparison with late-onset NODAT patients $(7.03 \pm 1.88 ; p=0.018)$. Multivariate analysis confirmed the significance of 'diabetogenic' allele number in the risk of developing late-onset NODAT (OR 1.37, $95 \%$ CI:1.05-1.78; $p=0.017$ ), which was also influenced by the occurrence of graft rejection episodes (Table 3 ). The sum of alleles was not associated with early-onset NODAT (OR $1.03,95 \%$ CI $0.83-1.27 ; p=0.801$ ), rather, steroid total dose was identified as a main independent risk factor. Additionally, when patients were classified according to the number of alleles previously associated with T2DM, those carrying more than a half of 'diabetogenic' alleles $(>7$ of the 14 alleles within 7 genes) were at significantly higher risk of NODAT (OR 2.17, 95 \% CI 1.18-3.99; $p=0.015$; Fig. 1).

\section{Discussion}

Both NODAT and T2DM share several risk factors (i.e., overweight or obesity, recipient age, Hispanic and AfricanAmerican ethnicity, CMV or HCV infection, age $>40$ years). Genetic factors may also play an important role, since a family history of T2DM among first-degree relatives has been identified as a NODAT risk factor [4]. In our study, we analyzed the potential effect of SNPs previously associated with T2DM on NODAT development in kidney transplant patients medicated with tacrolimus. An association between the SNPs investigated and T2DM was definitely confirmed, but their effect was relatively mild, with the odds ratios for most variants ranging from 1.10 to 1.20 , with a maximum of about 1.4 for TCF7L2 rs7903146 T. TCF7L2 rs7903146 T is the most prominent of all T2DM-associated common polymorphisms identified to date $[13,22]$. It is clear that one cannot detect the magnitude of the effects of these polymorphisms without analyzing thousands of patients. However, as the incidence of diabetes among transplant recipients medicated with tacrolimus is much higher than that in the general population, we had actually expected the influence of the investigated genetic variants on NODAT risk to be much more pronounced, as found by Yang et al. who observed an adjusted odds ratio value of $\geq 2$ for SNPs within HNF4A and IRS1 genes among Hispanic American kidney transplant recipients [17]. A study of Korean patients confirmed the association of eight SNPs in six genes (i.e., TCF7L2, SLC30A8, HHEX, CDKAL1, CDKN2A/B, and $K C N Q 1$ ) with NODAT in subjects medicated according to different immunosuppressive regimens (NODAT developed at different times during the observation period, with a median follow-up of $>10$ years) in a relatively large cohort of kidney transplant recipients. However, a long observation time might lead to an overlap of NODAT and T2DM in a later post-transplant period. The significance of analyzed variants could not be confirmed in our study, possibly due to the different ethnicity of the study participants, but also partly due to the limited number of patients analyzed, which is certainly one of the limitations of our study. The number of participants was limited as only patients receiving tacrolimus were included. Tacrolimus is known to increase the risk of NODAT in kidney allograft recipients compared to other immunosuppressants $[4,6]$. The majority of studies performed to date on the association of genetic factors with NODAT have included all patients regardless of immunosuppressive regimen $[17,19,23]$. In this respect, the group analyzed in our study is more homogenous, as all study participants were treated according to the same regimen (tacrolimus + mycofelate mofetil + steroids), which may translate into an increased sensitivity of the analysis.

Our investigation of the risk factors for early-onset NODAT (developed in the first 2 weeks post-transplantation) and lateonset NODAT revealed significant differences between these subgroups of patients. Total steroid dose, including methylprednisone administered as an induction of immunosuppressive therapy, significantly increased the risk of diabetes in the former group (early-onset) but not in the latter one. Indeed, mean steroid dose in the first week post-transplantation was significantly higher in early-onset NODAT patients (35.3 \pm $6.9 \mathrm{mg}$ /day prednisolone) than in patients without NODAT ( $31.5 \pm 8.5 \mathrm{mg} /$ day prednisolone; $p=0.017)$ as well as to the late-onset NODAT group $(31.3 \pm 7.7 \mathrm{mg} /$ day prednisolone; $p=$ 0.031 ). This observation may indicate that early-onset NODAT is triggered rather by steroids, while late-onset NODAT is 


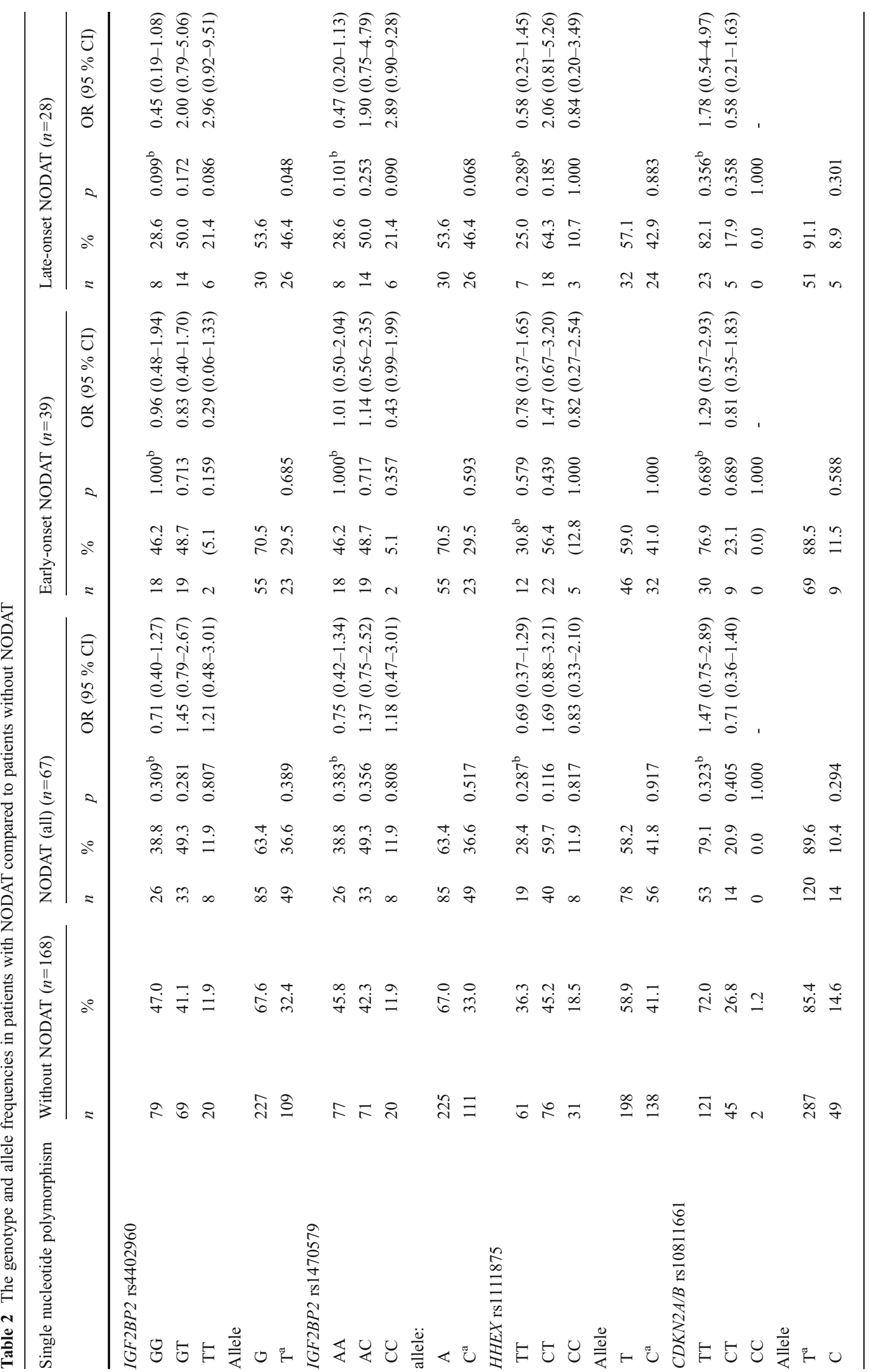




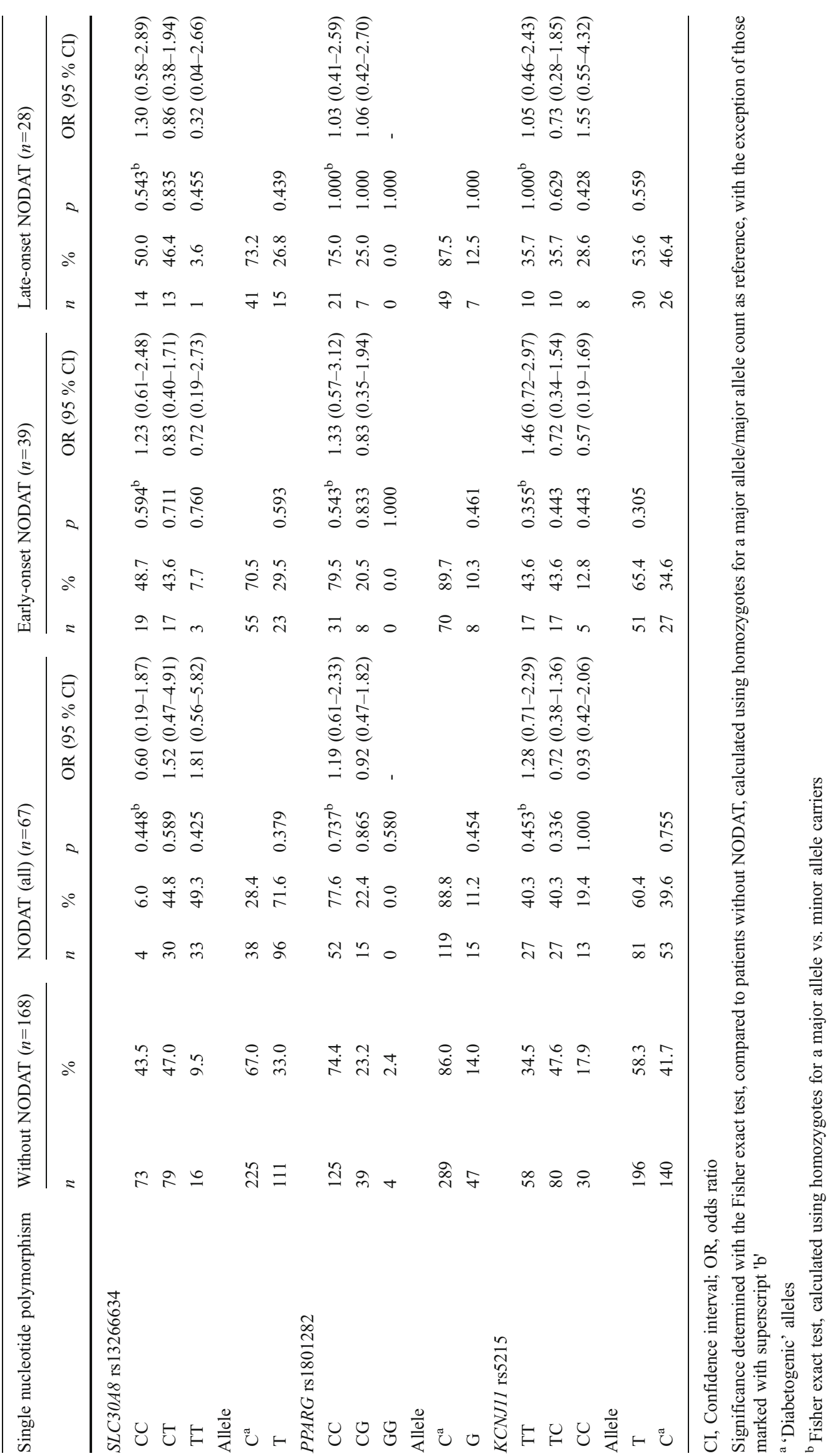


Table 3 Multivariate logistic regression analysis of potential risk factors for early-onset and late-onset NODAT

\begin{tabular}{|c|c|c|c|c|}
\hline \multirow[t]{2}{*}{ Independent variables } & \multicolumn{2}{|c|}{ Early NODAT $(n=39)$} & \multicolumn{2}{|c|}{ Late NODAT $(n=28)$} \\
\hline & OR $(95 \% \mathrm{CI})$ & $p$ & OR $(95 \% \mathrm{CI})$ & $p$ \\
\hline Patient's age & $1.02(0.99-1.05)$ & 0.181 & $1.02(0.99-1.06)$ & 0.206 \\
\hline BMI $\left(\mathrm{kg} / \mathrm{m}^{2}\right)$ & $1.05(0.95-1.16)$ & 0.315 & $1.11(0.99-1.24)$ & 0.074 \\
\hline Acute rejection episodes & $0.76(0.17-3.40)$ & 0.724 & $5.47(1.55-19.22)$ & 0.008 \\
\hline Steroid total dose $\mathrm{e}^{\mathrm{a}}$ & $2.40(1.05-5.51)$ & 0.037 & $0.93(0.50-1.72)$ & 0.801 \\
\hline Sum of the risk alleles ${ }^{b}$ & $1.03(0.83-1.27)$ & 0.801 & $1.37(1.05-1.78)$ & 0.017 \\
\hline
\end{tabular}

Early NODAT, up to 14 days post-transplantation; late-onset NODAT, developed later than 14 days post-transplantation

${ }^{\text {a }}$ Logarithmic transformation applied to fit normal distribution

${ }^{\mathrm{b}}$ Sum of alleles previously associated with T2DM: IGF2BP2 rs4402960 T, HHEX rs1111875 C, CDKN2A/B rs10811661 T, SLC30A8 rs13266634 C, PPARG rs $1801282 \mathrm{C}, K C N J 11$ rs5215 C, and TCF7L2 rs7903146 T

probably more dependent on other risk factors. The frequency of acute rejection episodes and total steroid dose are known to be associated with PTDM and are usually linked since high methylprednisolone doses (pulse therapy) are administered in the case of graft rejection. In our study, multivariate analysis revealed the occurrence of acute rejection episodes as an independent late-onset NODAT risk factor, while steroid dose

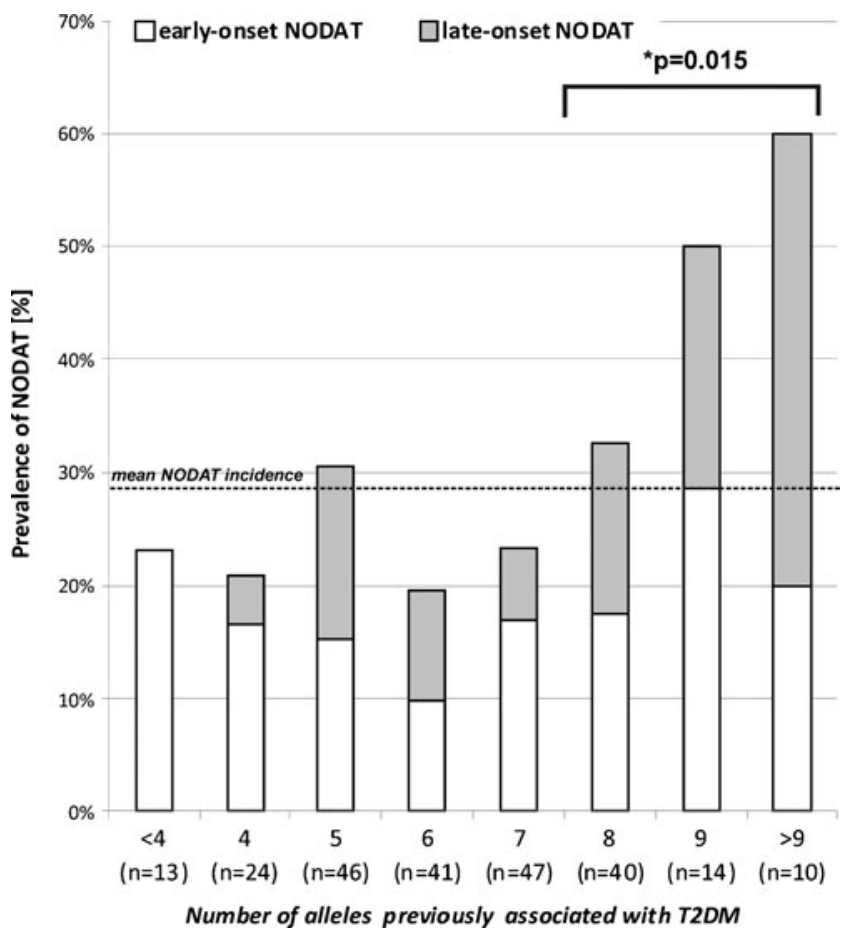

Fig. 1 Incidence of new-onset diabetes after transplantation (NODAT) in relation to the sum the alleles previously associated with type 2 diabetes mellitus (T2DM) (IGF2BP2 rs4402960 T, HHEX rs1111875 C, $C D K N 2 A / B$ rs10811661 T, SLC30A8 rs 13266634 C, PPARG rs $1801282 \mathrm{C}, K C N J 11$ rs5215 C, and TCF7L2 rs7903146 T). Asterisk indicates significance between patients inheriting $\leq 7$ vs. $>7$ alleles in relation to overall NODAT incidence, calculated by means of Fisher exact test (odds ratio 2.17, $95 \%$ confidence interval 1.18-3.99) was the only adjusted risk factor of early-onset NODAT. However, some patients received monoclonal antibodies instead of steroids during an acute rejection episode, which may explain the observed differences.

In order to investigate the cumulative effect of SNPs previously associated with T2DM on NODAT occurrence, we assessed the total number of 'diabetogenic' alleles inherited by each patient. Summing up the alleles of different genes would seem to be an acceptable approach since the effect of each SNP on T2DM risk is comparable $[13,22,24,25]$. Our analysis revealed that individuals carrying more than half of the total possible number of 'diabetogenic' alleles ( $>7$ of the 14 alleles of 7 genes) were at a significantly higher risk of NODAT, primarily due to an increased risk of late-onset NODAT in patients inheriting a greater number of 'risk' alleles. These findings were also confirmed in the multivariate analysis, where the sum of 'diabetogenic' alleles was revealed as a significant risk factor for late-onset-but not early-onset-NODAT. Based on the results of our study, we conclude that the effect of common polymorphisms in IGF2BP2, HHEX, CDKN2A/B, SLC30A8, $P P A R G$, and $K C N J 11$, all of which have been previously associated with T2DM, is not pronounced in the risk of NODAT in kidney transplant recipients treated with tacrolimus. However, the sum of 'diabetogenic' alleles may be one of the factors increasing the incidence of post-transplant diabetes, especially in the case of lateonset NODAT, which seems to be less dependent on steroid treatment. Our results support the observation that a complex analysis of diabetes risk genotypes may lead to the identification of disease susceptibility predictors, also for NODAT, and that this approach may have an advantage over single-locus studies [26]. Finally, the application of the analyzed genetic markers in therapy individualization should be verified by further independent studies in populations of different ethnicities. 
Open Access This article is distributed under the terms of the Creative Commons Attribution License which permits any use, distribution, and reproduction in any medium, provided the original author(s) and the source are credited.

\section{References}

1. Räkel A, Karelis AD (2011) New-onset diabetes after transplantation: risk factors and clinical impact. Diabetes Metab 37:1-14

2. Davidson J, Wilkinson A, Dantal J, Dotta F, Haller H, Hernández D, Kasiske BL, Kiberd B, Krentz A, Legendre C, Marchetti P, Markell M, van der Woude FJ, Wheeler DC (2003) International Expert Panel. New-onset diabetes after transplantation: 2003 International consensus guidelines. In: Proc Int expert panel meeting. Transplantation 75:SS3-SS24

3. Montori VM, Basu A, Erwin PJ, Velosa JA, Gabriel SE, Kudva YC (2002) Posttransplantation diabetes: a systematic review of the literature. Diabetes Care 25:583-592

4. Kasiske BL, Snyder JJ, Gilbertson D, Matas AJ (2003) Diabetes mellitus after kidney transplantation in the United States. Am J Transplant 3:178-185

5. van Hooff JP, Christiaans MH, van Duijnhoven EM (2005) Tacrolimus and posttransplant diabetes mellitus in renal transplantation. Transplantation 79:1465-1469

6. Vincenti F, Friman S, Scheuermann E, Rostaing L, Jenssen T, Campistol JM, Uchida K, Pescovitz MD, Marchetti P, Tuncer M, Citterio F, Wiecek A, Chadban S, El-Shahawy M, Budde K, Goto N (2007) DIRECT (Diabetes Incidence after Renal Transplantation: Neoral C Monitoring Versus Tacrolimus) Investigators. Results of an international, randomized trial comparing glucose metabolism disorders and outcome with cyclosporine versus tacrolimus. Am J Transplant 7:1506-1514

7. Tamura K, Fujimura T, Tsutsumi T, Nakamura K, Ogawa T, Atumaru C, Hirano Y, Ohara K, Ohtsuka K, Shimomura K et al (1995) Transcriptional inhibition of insulin by FK506 and possible involvement of FK506 binding protein-12 in pancreatic beta-cell. Transplantation 59:1606-1613

8. Weir MR, Fink JC (1999) Risk for posttransplant diabetes mellitus with current immunosuppressive medications. Am J Kidney Dis 34:1-13

9. Nam JH, Mun JI, Kim SI, Kang SW, Choi KH, Park K, Ahn CW, Cha BS, Song YD, Lim SK, Kim KR, Lee HC, Huh KB (2001) beta-Cell dysfunction rather than insulin resistance is the main contributing factor for the development of postrenal transplantation diabetes mellitus. Transplantation 71:1417-1423

10. Sladek R, Rocheleau G, Rung J, Dina C, Shen L, Serre D et al (2007) A genome-wide association study identifies novel risk loci for type 2 diabetes. Nature 445:881-885

11. Saxena R, Voight BF, Lyssenko V, Burtt NP, de Bakker PI, Chen H et al (2007) Genome-wide association analysis identifies loci for type 2 diabetes and triglyceride levels. Science 316:1331-1336

12. Scott LJ, Mohlke KL, Bonnycastle LL, Willer CJ, Li Y, Duren WL et al (2007) A genome-wide association study of type 2 diabetes in Finns detects multiple susceptibility variants. Science 316:1341-1345

13. Zeggini E, Weedon MN, Lindgren CM, Frayling TM, Elliott KS, Lango $\mathrm{H}$ et al (2007) Replication of genome-wide association signals in UK samples reveals risk loci for type 2 diabetes. Science 316:1336-1341
14. Takeuchi F, Serizawa M, Yamamoto K, Fujisawa T, Nakashima E, Ohnaka $\mathrm{K}$ et al (2009) Confirmation of multiple risk Loci and genetic impacts by a genome-wide association study of type 2 diabetes in the Japanese population. Diabetes 58(7):1690-1699

15. Billings LK, Florez JC (2010) The genetics of type 2 diabetes: what have we learned from GWAS? Ann N Y Acad Sci 1212:5977

16. Tavira B, Coto E, Díaz-Corte C, Ortega F, Arias M, Torres A, Díaz JM, Selgas R, López-Larrea C, Campistol JM, Ruiz-Ortega M, Alvarez V (2011) Pharmacogenetics of Tacrolimus REDINREN Study Group. KCNQ1 gene variants and risk of new-onset diabetes in tacrolimus-treated renal-transplanted patients. Clin Transplant 25:E284-E291

17. Yang J, Hutchinson II, Shah T, Min DI (2011) Genetic and clinical risk factors of new-onset diabetes after transplantation in Hispanic kidney transplant recipients. Transplantation 91:1114-1119

18. Kurzawski M, Dziewanowski K, Kedzierska K, Gornik W, Banas A, Drozdzik M (2010) Association of calpain-10 gene polymorphism and posttransplant diabetes mellitus in kidney transplant patients medicated with tacrolimus. Pharmacogenomics J 10:120-125

19. Kang ES, Kim MS, Kim CH, Nam CM, Han SJ, Hur KY, Ahn CW, Cha BS, Kim SI, Lee HC, Kim YS (2009) Association of common type 2 diabetes risk gene variants and posttransplantation diabetes mellitus in renal allograft recipients in Korea. Transplantation 88:693-698

20. Kurzawski M, Dziewanowski K, Kędzierska K, Wajda A, Lapczuk J, Droździk M (2011) Association of transcription factor 7-like 2 (TCF7L2) gene polymorphism with posttransplant diabetes mellitus in kidney transplant patients medicated with tacrolimus. Pharmacol Rep 63:826-833

21. Grant SF, Thorleifsson G, Reynisdottir I, Benediktsson R, Manolescu A, Sainz J, Helgason A, Stefansson H, Emilsson V, Helgadottir A, Styrkarsdottir U, Magnusson KP, Walters GB, Palsdottir E, Jonsdottir T, Gudmundsdottir T, Gylfason A, Saemundsdottir J, Wilensky RL, Reilly MP, Rader DJ, Bagger Y, Christiansen C, Gudnason V, Sigurdsson G, Thorsteinsdottir U, Gulcher JR, Kong A, Stefansson K (2006) Variant of transcription factor 7-like 2 (TCF7L2) gene confers risk of type 2 diabetes. Nat Genet 38:320-323

22. Diabetes Genetics Initiative of Broad Institute of Harvard and MIT, Lund University, and Novartis Institutes of BioMedical Research, Saxena R, Voight BF, Lyssenko V, Burtt NP, de Bakker PI, Chen H et al (2007) Genome-wide association analysis identifies loci for type 2 diabetes and triglyceride levels. Science 316:1331-1336

23. Bamoulid J, Courivaud C, Deschamps M, Mercier P, Ferrand C, Penfornis A, Tiberghien P, Chalopin JM, Saas P, Ducloux D (2006) IL-6 promoter polymorphism -174 is associated with new-onset diabetes after transplantation. J Am Soc Nephrol 17:2333-2340

24. Zeggini E, Scott LJ, Saxena R, Voight BF, Marchini JL, Hu T et al (2008) Meta-analysis of genome-wide association data and largescale replication identifies additional susceptibility loci for type 2 diabetes. Nat Genet 40:638-645

25. Voight BF, Scott LJ, Steinthorsdottir V, Morris AP, Dina C, Welch RP et al (2010) Twelve type 2 diabetes susceptibility loci identified through large-scale association analysis. Nat Genet 42:579-589

26. den Hoed M, Ekelund U, Brage S, Grontved A, Zhao JH, Sharp SJ, Ong KK, Wareham NJ, Loos RJ (2010) Genetic susceptibility to obesity and related traits in childhood and adolescence: influence of loci identified by genome-wide association studies. Diabetes 59 (11):2980-2988 